SHORT REPORT

\title{
New insights into the glucose oxidase stick test for cerebrospinal fluid rhinorrhoea
}

\author{
E H Baker, D M Wood, A L Brennan, D L Baines, B J Philips
}

Emerg Med J 2005;22:556-557. doi: 10.1136/emj.2004.022111

Rhinorrhoea is a clinical sign of cerebrospinal fluid (CSF) leakage in patients with skull fracture, but can also be attributable to respiratory secretions or tears. Laboratory tests confirming the presence of CSF are not sufficiently rapid to support clinical decision making in the emergency department and may not be universally available.

Detection of glucose in nasal discharge was traditionally used to diagnose CSF leak at the bedside, but has fallen into disuse as it has poor positive predictive value. We propose an algorithm to improve the diagnostic value of this test taking into consideration factors we have found to affect the glucose concentration of respiratory secretions. In patients at risk of CSF leak, nasal discharge is likely to contain CSF if glucose is present in the absence of visible blood, if blood glucose is $<6 \mathrm{mmol} . \mathrm{L}^{-1}$, and if there are no symptoms of upper respiratory tract infection.

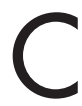
erebrospinal fluid (CSF) leakage from dural tears complicates around $20 \%$ of basilar skull fractures and $25 \%$ of facial fractures. ${ }^{12}$ As CSF leakage is commonly complicated by intracranial infection in $3-31 \%$ cases, ${ }^{13}{ }^{4}$ it is important not to miss the diagnosis. Clinical suspicion of CSF leak should be raised by the presence of rhinorrhoea; however, differentiating CSF from other causes of nasal discharge (respiratory secretions, tears, and blood) presents a diagnostic challenge. Immunofixation electrophoresis of nasal secretions in the laboratory can be used to detect $\beta$-2transferrin-a protein produced by neuraminidase activity in the brain and uniquely found in CSF and perilymph. ${ }^{5}$ This test is not sufficiently rapid to provide support for clinical decision making in emergency departments and may not be available in all hospitals, particularly in developing countries.

Measurement of the glucose concentration of nasal discharge is a traditional bedside test for the detection of CSF leaks. Glucose oxidase sticks are wetted with nasal secretions and colour change is compared with a calibrated scale to determine glucose concentration. The presence of glucose is taken to indicate that secretions contain CSF. This test is easy to perform, cheap, and widely available, but has poor positive predictive value for CSF leakage. Glucose was detected in nasal discharge from 15/17 normal children ${ }^{6}$ and in $44 \%$ of clear nasal and lacrimal secretions from people without any risk factor for CSF leak. ${ }^{7}$ False negative results, where glucose oxidase sticks have failed to detect CSF, have also been reported. ${ }^{5}{ }^{8}$ We propose that if factors determining the appearance of glucose in respiratory secretions can be explained, this old test could still have a role in identification of CSF leaks.

\section{FACTORS DETERMINING THE GLUCOSE} CONCENTRATION OF RESPIRATORY SECRETIONS

We have adapted the glucose oxidase stick technique to measure the glucose concentration of nasal secretions in the absence or presence of rhinorrhoea. ${ }^{9}$ Glucose oxidase sticks
(Roche Diagnostics, UK) were placed in contact with the nasal mucosa under direct vision using an otoscope and nasal secretions allowed to wet the glucose oxidase for 30 seconds. On removal, coating of the stick by secretions was verified by inspection. The stick was wiped after 30 seconds and read against a visual colour scale after a further minute. Using this technique we found that glucose was not detectable in nasal secretions from 20 healthy volunteers with normal nasal mucosa and normal blood glucose. ${ }^{9}$ Glucose was, however, present in nasal secretions of $12 / 24$ people with nasal inflammation due to viral colds at $1-2$ mmol. $L^{-1}{ }^{9}$ Ten of the people with detectable nasal glucose were re-tested two weeks later when rhinitis had resolved and nine of these no longer had glucose in nasal secretions. The person who still had detectable nasal glucose had persistent coryzal symptoms. Nasal epithelial inflammation induced experimentally by five or more repeat insertions of glucose oxidase sticks also increased the glucose concentration of nasal secretions from undetectable to $0.9 \pm 0.5 \mathrm{mmol} . \mathrm{L}^{-1}$ in healthy volunteers. ${ }^{10}$

The glucose concentration of nasal secretions is also increased where blood glucose is raised. Eighteen of 20 people with diabetes mellitus attending an out-patient clinic had 4 (2-7) mmol.L ${ }^{-1}$ (median (interquartile range)) glucose in airway secretions. ${ }^{9}$ We have described the relation between blood and nasal glucose concentrations in healthy people in more detail using a modified hyperglycaemic clamp technique. ${ }^{10}$ Blood glucose was raised in volunteers by controlled infusion of $20 \%$ dextrose and nasal glucose measured at predetermined blood glucose concentrations. Glucose was detected in nasal secretions above a blood glucose concentration of $6.7-9.7 \mathrm{mmol} . \mathrm{L}^{-1}$ in each of 12 volunteers. Nasal glucose concentration changed within 10 minutes of a change in blood glucose concentration and glucose was removed from nasal secretions if blood glucose was allowed to fall. A $75 \mathrm{~g}$ oral glucose load also increased blood glucose to $10-11 \mathrm{mmol} . \mathrm{L}^{-1}$, which was sufficient for glucose to become detectable in nasal secretions. ${ }^{10}$

\section{ACCURACY OF GLUCOSE OXIDASE STICKS IN THE MEASUREMENT OF GLUCOSE CONCENTRATION IN RESPIRATORY SECRETIONS AND CSF}

Glucose oxidase sticks are designed to measure glucose concentrations in blood and hence function optimally at concentrations around normal blood glucose values $\left(\sim 4\right.$ mmol. $\left.\mathrm{L}^{-1}\right) .^{5}$ We compared glucose measurements obtained by glucose oxidase sticks with values obtained by a glucose analyser (GM9D, Analox Instruments, London, UK) in 50 samples of lower airway secretions obtained from patients intubated on intensive care. ${ }^{9}$ Lower airway secretions were both tested with glucose oxidase sticks and filtered to remove particulate matter before analysis of the filtrate $(0.45 \mu \mathrm{m}$, Sartorius, Goettingen, Germany). Glucose measurements by sticks and analyser were similar where the glucose concentration of airway secretions was

Abbreviations: CSF, cerebrospinal fluid 


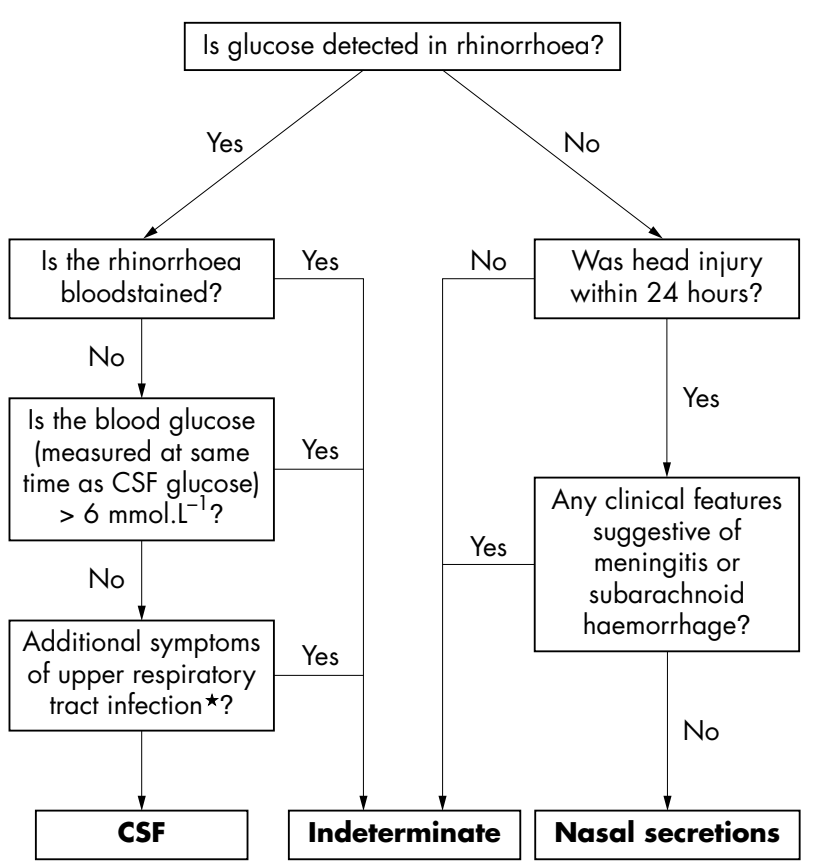

$\star$ These include sneezing, nasal blockage, cough, sore throat, sputum or purulent nasal discharge

Figure 1 Algorithm to assist in evaluation of rhinorrhoea following a head injury

1-4 mmol. $\mathrm{L}^{-1}$. At higher glucose concentrations the sticks tended to overestimate the glucose concentration of airway secretions compared with the analyser. There were five false negative readings where the sticks did not detect glucose of 0.7-2.0 mmol. $\mathrm{L}^{-1}$ measured by the analyser.

In a previous study, glucose oxidase sticks from four different manufacturers (Visidex IIR, Haemo-Glukotest 20800RR, Multistix R, and DextrostixR) were used to determine the glucose concentrations of $54 \mathrm{CSF}$ samples. ${ }^{11}$ The results were compared with a standard glucose oxidase method (Beckman). The closest correlation between the standard method and glucose oxidase sticks was found for the DextrostixR when used with a glucometer $(r=0.8002)$. All four methods detected two cases with CSF glucose $<1.5 \mathrm{mmol} . \mathrm{L}^{-1}$, but did not produce clear results for three cases with CSF glucose values $2.0-2.2 \mathrm{mmol} . \mathrm{L}^{-1}$. The authors commented that these results were confusing, and the implication is that glucose oxidase sticks are inaccurate at low CSF glucose concentrations.

\section{IMPLICATIONS FOR USE OF GLUCOSE MEASUREMENTS TO DETECT CSF LEAK}

We propose a simple algorithm to optimise the use of glucose measurement for the bedside detection of CSF leak in the emergency department (fig l). If rhinorrhoea contains glucose, the specificity of the test for CSF can be improved by excluding other factors that increase the glucose concentration of nasal discharge. If the nasal discharge is not blood stained, the blood glucose (measured at the same time as CSF glucose) is $<6 \mathrm{mmol} . \mathrm{L}^{-1}$, and there are no other symptoms of upper respiratory tract infection, such as sneezing, nasal blockage, cough, sore throat, sputum, or purulent nasal discharge, then this increases the likelihood that the discharge contains CSF. If rhinorrhoea does not contain glucose, then either it does not contain CSF or CSF glucose concentrations are below the limit of detection by the sticks (false negative measurement). CSF glucose concentrations may be low in people with bacterial meningitis, subarachnoid haemorrhage, neoplasia, and neurological complications of connective tissue disease. ${ }^{12}$ As both bacterial meningitis and subarachnoid haemorrhage ${ }^{13}$ may complicate head trauma, these should be considered in patients with glucose-free rhinorrhoea before ruling out a CSF leak. In people with recent head injury ( $<24$ hours) who do not have blood stained nasal discharge or clinical features suggestive of meningitis or subarachnoid haemorrhage, it is likely that glucose free secretions do not contain CSF.

\section{COMMENT}

Detection of glucose in rhinorrhoea cannot be used alone to diagnose CSF leak nor can absence of glucose always exclude CSF leak. However, we anticipate that modified glucose testing, as proposed in our algorithm, could support clinical decision making, particularly in situations where more complex tests for CSF leak are not available.

\section{Authors' affiliations}

E H Baker, D M Wood, A L Brennan, D L Baines, B J Philips, Glucose and Pulmonary Infection Group, St George's, University of London, London, UK

\section{Funding: none.}

\section{Competing interests: none declared}

Statement: The Corresponding Author has the right to grant on behalf of all authors and does grant on behalf of all authors, an exclusive licence (or non exclusive for government employees) on a worldwide basis to the BMJ Publishing Group Ltd and its Licensees to permit this article (if accepted) to be published in EMJ editions and any other BMJPG Ltd products to exploit all subsidiary rights, as set out in our licence.

Ethical approval: approval obtained.

Correspondence to: Dr Emma Baker, Reader in Clinical Pharmacology, Cardiac and Vascular Sciences, Physiological Medicine, St George's, University of London, Cranmer Terrace, London, SW17 ORE, UK; ebaker@sgul.ac.uk

Accepted for publication 22 December 2004

\section{REFERENCES}

1 Dagi TF, Meyer FB, Poletti CA. The incidence and prevention of meningitis after basilar skull fracture. Am J Emerg Med 1983;1:295-8.

2 Eljamel MS. Fractures of the middle third of the face and cerebrospinal fluid rhinorrhoea. Br J Neurosurg 1994;8:289-93.

3 Clemenza JW, Kaltman SI, Diamond DL. Craniofacial trauma and cerebrospinal fluid leakage: a retrospective clinical study. J Oral Maxillofac Surg 1995;53:1004-7.

4 Eljamel MS, Foy PM. Acute traumatic CSF fistulae: the risk of intracranial infection. Br J Neurosurg 1990;4:381-5.

5 Chan DTM, Poon WS, Ip CP, et al. How useful is glucose detection in diagnosing cerebrospinal fluid leak? The rational use of CT and beta- 2 transferrin assay in detection of cerebrospinal fluid fistula. Asian J Surg 2004;27:39-42.

6 Hull HF, Morrow G. Glucorrhoea revisited. Prolonged promulgation of another plastic pearl. JAMA 1975;234:1052-3.

7 Steedman DJ, Gordon M. CSF rhinorrhoea: significance of the glucose oxidase strip test. Injury 1987;18:327-8.

8 Katz RT, Kaplan PE. Glucose oxidase sticks and cerebrospinal fluid rhinorrhoea. Arch Phys Med Rehabil 1985;66:391-3.

9 Philips BJ, Meguer JX, Redman J, et al. Factors determining the appearance of glucose in upper and lower respiratory tract secretions. Intens Care Med 2003;29:2204-10.

10 Wood DM, Brennan AL, Philips BJ, et al. Effect of hyperglycaemia on glucose concentration of airways secretions. Clin Sci 2004;106:527-33.

11 Muller PD, Donald PR. Reagent strips in the evaluation of cerebrospinal fluid glucose levels. Ann Trop Paediatr 1987;7:287-9.

12 Fishman RA. Cerebrospinal fluid in diseases of the nervous system, $2^{\text {nd }}$ edition. Philadelphia, PA: WB Saunders Co, 1992.

13 Mattioli C, Beretta L, Gerevini S, et al. Traumatic subarachnoid hemorrhage on the computerized tomography scan obtained at admission: a multicenter assessment of the accuracy of diagnosis and the potential impact on patient outcome. J Neurosurg 2003;98:37-42. 